\title{
Schwierige Entscheidungen am Lebensende
}

\section{Hans-Georg Bone}

ich wollt noch danke sagen

doch ich lieg im krankenwagen

noch wolln sie mich zwangsbeatmen

doch bald is alles aus und vorbei

Liedtextauszug aus „Danke“ - Die Fantastischen Vier

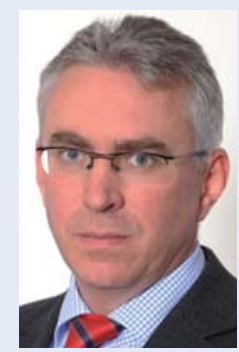

Hans-Georg Bone

A schen Vier spricht die Angst, dass man vor dem Tod noch gegen seinen Willen, i.d.R. an denen man vor wenigen Jahrzehnten noch sicher versterben musste, können mit Hilfe der modernen Intensivmedizin und ihrer Überbrückungsverfahren
Korrespondenzadresse

Prof. Dr. med.

Hans-Georg Bone Klinikum Vest GmbH Klinik f. Anästhesiologie u. Intensivmedizin Knappschaftskrankenhaus

Dorstener Straße 151 45657 Recklinghausen Bone.Hans-Georg@ KK-Recklinghausen.de us den obigen Zeilen des Liedes der Fantastiauch auf einer Intensivstation, „zwangsbeatmet" wird. In den letzten Jahrzehnten hat die Intensivmedizin enorme Fortschritte gemacht. Erkrankungen, für versagende Organsysteme, wie z.B. Beatmung, Nierenersatzverfahren oder auch extrakorporale kardiale oder pulmonale Unterstützungssysteme, überlebt werden. Gleichzeitig liegt im Erfolg der modernen Intensivmedizin aber auch ihre Gefahr, nämlich einen unvermeidbaren Tod ggf. nur weiter hinauszuzögern oder aber zwar ein Überleben zu ermöglichen, aber den Patienten in einer Weise überleben zu lassen, die er selbst nicht gewollt hätte. Betrachtet man die Statistiken, so ist ein Versterben auf einer Intensivstation mit einer Maximaltherapie bis zum Tode eher eine Ausnahme. In der ETHICUS-Studie wurden auf 37 Intensivstationen in 17 europäischen Ländern im Jahr 2000 untersucht, ob und wie Intensivpatienten versterben [1]. Ca. 13\% aller Intensivpatienten verstarben in diesem Zeitraum auf der Intensivstation. Bei 76\% dieser Patienten ging dem Versterben eine Therapiereduktion oder ein Therapieverzicht voraus [1]. Bei der Befragung von Ärzten auf deutschen kardiochirugischen Intensivstationen wurden als medizinische Gründe für eine Therapiereduktion bzw. einen Therapieabbruch genannt: schlechte Prognose auf Grund eines craniales Computertomogramms, ein Multiorganversagen und das Versagen von Assist-Devices [2]. Als ethische Gründe für eine Therapiereduktion bzw. einen Therapieabbruch wurden genannt: erwartete schlechte Lebensqualität des Patienten, Wunsch des Patienten und Entscheidung der Angehörigen [2]. Oben erwähnte Gründe für eine Therapiereduktion, die infauste Prognose und der Wille des Patienten gegen eine weitere Therapie kommen auch in den Grundsätzen der Bundesärztekammer zur Sterbebegleitung zum Ausdruck [3]:

„Ein offensichtlicher Sterbevorgang soll nicht durch lebenserhaltende Therapien künstlich in die Länge gezogen werden. Darüber hinaus darf das Sterben durch Unterlassen, Begrenzen oder Beenden einer begonnenen medizinischen Behandlung ermöglicht werden, wenn dies dem Willen des Patienten entspricht."

Obwohl innerhalb der letzten 10 Jahre viel über die medizinischen, rechtlichen und ethischen Grundlagen der Therapiereduktion auf Intensivstationen gesprochen und geschrieben wurde, existieren weiterhin viele Ängste in der Bevölkerung und bei den Patienten, aber auch Unsicherheiten bei den auf den Intensivstation arbeitenden Ärzten und Pflegekräften. Die in dieser Ausgabe veröffentlichte Arbeit von Professor Prien soll diese Unsicherheiten der Intensivärzte und Pflegekräfte reduzieren. Neben einer kurzen Darstellung der rechtlichen und ethischen Grundlagen einer Therapiereduktion oder besser einer Therapiezieländerung gibt Prien den Lesern anhand von 5 Fragen Hilfen zur Lösung der konkreten Probleme am Bett des Intensivpatienten:

1. Ist eine Maßnahme medizinisch indiziert?

2. Ist der Patient entscheidungsfähig?

3. Liegt eine verbindliche Patientenverfügung vor?

4. Ist eine Betreuung eingerichtet oder liegt eine Vorsorgevollmacht vor?

5. Was hätte der Patient mutmaßlich gewollt?

Wie schwer dennoch Entscheidungen bezüglich Therapieverzicht oder Therapiereduktion sein können, zeigt sich an einem prominenten Beispiel:

Der weltberühmte Herz- und Thoraxchirurg Prof. Dr. Michael E. DeBakey erkrankte plötzlich am 31. Dezember 2005, nachdem er, trotz seines hohen Alters von 96 Jahren, ein sehr aktives Leben geführt hatte. Es wurde eine Dissektion der Aorta ascendens diagnosti- 
ziert, eine Erkrankung, die nach DeBakey klassifiziert wird und deren chirurgische Behandlung er maßgeblich entwickelt hat. Trotz vielfacher Gespräche und Befragung wurde nicht klar, ob DeBakey operiert werden wolle oder nicht. Der Herzchirurg, einer seiner ehemaligen Schüler, entschied sich dann zur Operation. Nach der Operation mit nachfolgenden Komplikationen und zögerlicher Rekonvaleszenz äußerte DeBakey sich glücklich darüber, operiert und vor dem sicheren Tod gerettet worden zu sein [4]. Bei den schwierigen Entscheidungen am Lebenende sollte auch mit berücksichtigt werden, wie unterschiedlich die Sicht zwischen den auf den Intensivstationen arbeitenden und den Intensivpatienten und ihren Angehörigen sein kann. In der ETHICATT-Studie wurden in 6 europäischen Ländern Intensivärzte, Intensivpflegekräfte, ehemalige Intensivpatienten und deren Angehörige befragt [5]. Während bei den Ärzten $88 \%$ und bei den Pflegekräften $87 \%$ die Lebensqualität für wichtiger erachteten als das Leben an sich, war das Ergebnis bei den ehemaligen Patienten mit 51\% keineswegs so eindeutig. Über 50\% der ehemaligen Patienten würden für sich eine Reanimation wünschen, selbst bei unheilbarer Grunderkrankung [5]. Diese Ergebnisse zeigen, dass man es sich als Intensivarzt oder -pflegekraft nicht leicht machen sollte mit den Entscheidungen über die einem anvertrauten Patienten. Arbeiten wie die von Professor
Prien oder auch das neue Positionspapier der Sektion Ethik der Deutschen Interdisziplinären Vereinigung für Intensiv- und Notfallmedizin [6] können bei diesen Entscheidungen helfen. Hoffentlich sinkt nach und nach auch langsam die Angst einiger Zeitgenossen, auf Intensivstationen „zwangsbeatmet“ zu werden.

\section{Literatur}

1 Sprung CL, Cohen SL, Sjokvist P et al. End-of-life practices in European intensive care units: the Ethicus Study. JAMA 2003; 290: $790-797$

2 Schimmer C, Gorski A, Ozkur M et al. Policies of withholding and withdrawal of life-sustaining treatment in critically ill patients on cardiac intensive care units in Germany: a national survey. Interact Cardiovasc Thorac Surg 2012; 14: 294-299

3 Bundesärztekammer. Grundsätze der Bundesärztekammer zur ärztlichen Sterbebegleitung. Dtsch Arztebl 2011; 108: A-346-348

4 Gaul C, Helm J. Entscheidungen am Lebensende „Hochkomplex und individuell“. Dtsch Arztebl 2009; 106: A-84

5 Sprung CL, Carmel S, Sjokvist P et al. Attitudes of European physicians, nurses, patients, and families regarding end-of-life decisions: the ETHICATT study. Intensive Care Med 2007; 33: $104-110$

6 Janssens U, Burchardi H, Duttge $\mathrm{G}$ et al. Therapiezieländerung und Therapiebegrenzung in der Intensivmedizin: Positionspapier der Sektion Ethik der Deutschen Interdisziplinären Vereinigung fur Intensiv- und Notfallmedizin. Anaesthesist 2013; 62: $47-52$ 